\title{
AN EFFICIENT METHOD TO SURVEY TYPICAL OBJECTS IN NEARBY GALAXIES
}

\author{
G. MURATORIO and M. AZZOPARDI \\ Observatoire de Marseille \\ 2 Place Le Verrier \\ F-13248 Marseille Cedex 4 \\ France
}

Within the framework of our studies of the stellar populations in nearby galaxies (Azzopardi 1984, 1993, and references therein), we have shown for some time that slitless spectroscopy, through either transmission gratings (grisms/grens) or prisms in combination with suitable intermediate band-pass interference filters, allows the detection of a number of objects with outstanding spectral characteristics, even in very crowded fields. We are now carrying out very deep slitless spectroscopy surveys in selected areas of nearby galaxies using both the ESO Faint Object Spectrograph and Camera (EFOSC) devices attached at the Cassegrain foci of either the ESO $3.6 \mathrm{~m}$ or the Max Plank Gesellschaft/ESO $2.2 \mathrm{~m}$ telescopes, as well as the ESO Multi-Mode
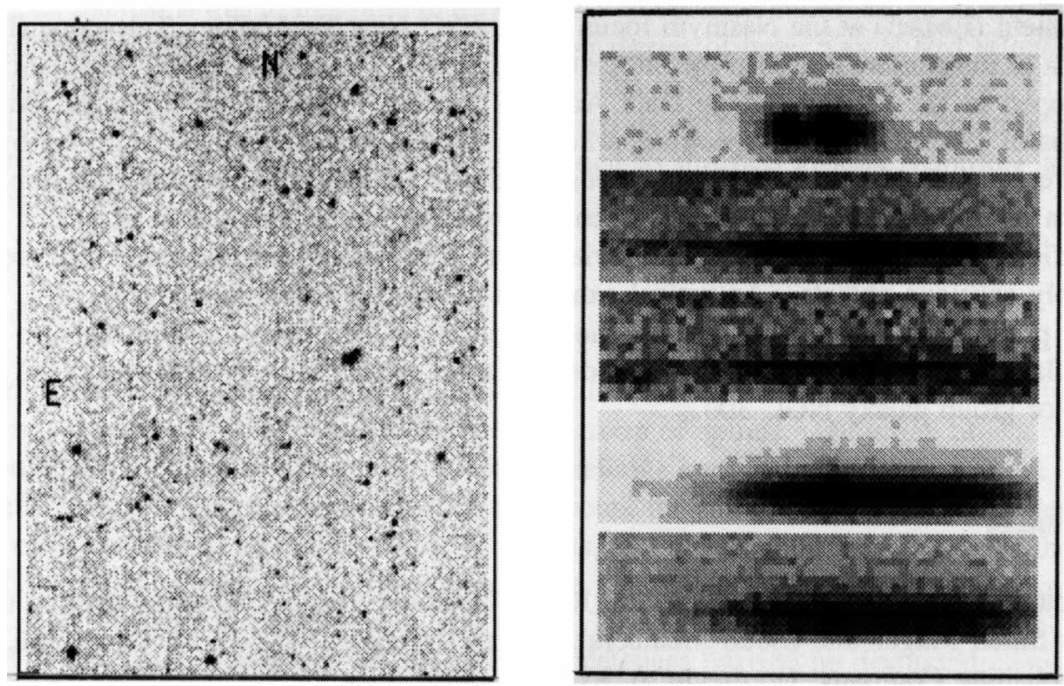

Figure 1. LMC field $\left(3.6^{\prime} \times 4.2^{\prime}\right)$ obtained with EFOSC in the direct imaging mode at the ESO $3.6 \mathrm{~m}$ telescope. Five $\mathrm{H} \alpha$ emission-line objects have been detected. Their spectra have been extracted from the respective slitless spectroscopic frame secured with the $\mathrm{R} 300$ grism $(270 \AA / \mathrm{mm}, 3.5 \AA /$ px dispersion) and through an interference filter $(\mathrm{CW}=6569 \AA, \mathrm{BW}=110 \AA)$. The first one, which exhibits the $\mathrm{H \alpha}+\lambda 6548$ - 6583 NII emission-lines, is the faint planetary nebula No. 48 identified in the meantime by Morgan \& Good (1992). 

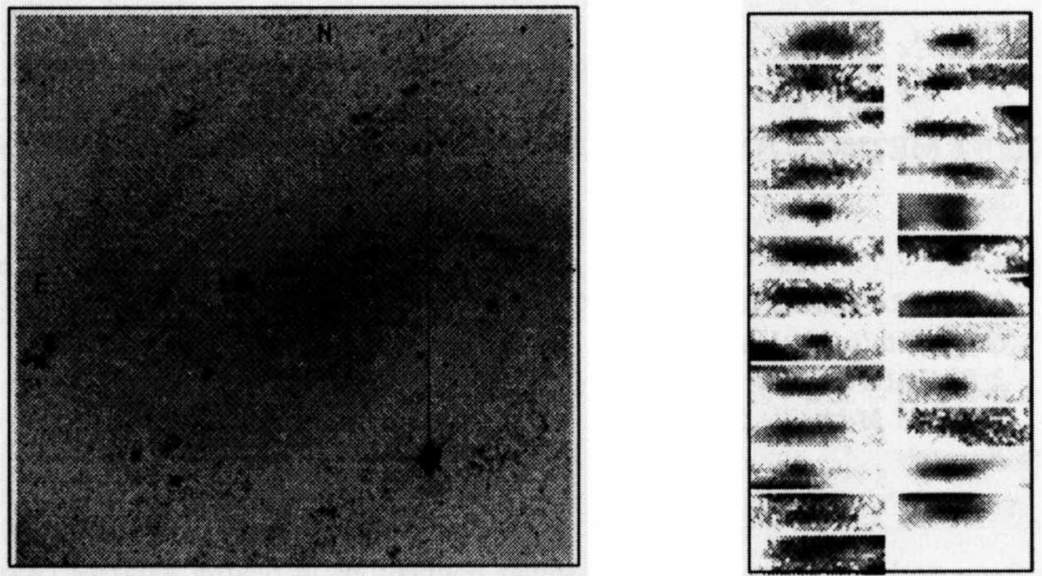

Figure 2. NGC 300 field $\left(7.6^{\prime} \times 7.6^{\prime}\right)$ obtained with EMMI in the direct imaging mode at the ESO $3.5 \mathrm{~m}$ NTT. The WR are searched for using either the $\lambda 4686 \mathrm{HeII}$ or $\lambda 4650 \mathrm{CIII}$ emission lines. 25 very faint WR star candidates have been detected. Their spectra have been extracted from the respective slitless spectroscopic frame secured with a grism yielding a dispersion of $515 \AA / \mathrm{mm}(9.7 \AA / \mathrm{px})$ through an interference filter $(\mathrm{CW}=4692 \AA, \mathrm{BW}=144 \AA)$. The nature of some of them was previously confirmed by Schild \& Testor (1992).

Instrument (EMMI) at the Nasmyth focus of the ESO New Technology Telescope (NTT). The spectra of interest are searched for on the CCD frames by means of a semi-automatic procedure developed in the Munich Image Data Analysis System (MIDAS) environment (Muratorio \& Azzopardi 1992). Each 2D-spectrum, whose position is automatically determined by means of the direct imaging frame, is background corrected, normalized and compared with some reference spectra of objects whose nature has been firmly established. All the objects, that have been identified through impersonal selection criteria, are then visually scrutinized in order to reject any possibly spurious objects. As typical examples of our field spectroscopy surveys, we present some preliminary results concerning the detection of $\mathrm{H} \alpha$ emission-line stars in the Large Magellanic Cloud (LMC) as well as the identification of very faint Wolf-Rayet (WR) stars in the Sculptor group galaxy NGC 300.

\section{References}

Azzopardi, M., 1984. 'Narrow Spectral Range Objective-Prism Technique applied to a Search for Small Magellanic Cloud Members', in Proc. IAU Coll. 78, ed. M. Capaccioli, D. Reidel Publishing Company, Dordrecht, p. 351.

Azzopardi, M. 1993. 'Survey of Carbon Stars and Emission Objects', in Proc. 2nd European Meeting on the Magellanic Clouds, eds. B. Baschek, G. Klare and J. Lequeux, SpringerVerlag, Berlin, p. 86.

Morgan, D.H. and Good, A.R., 1992. Astron. Astrophys. Suppl., 92, 571.

Muratorio, G. and Azzopardi, M., 1992. 'Reduction of Slitless Spectroscopy Data', in ESO Conference and Workshop Proc. No. 41, eds. P.J. Grosbøl and R.C.E. de Ruijsscher, p. 35.

Schild, H. and Testor, G., 1992. Astron. Astrophys., 266, 145. 